\title{
Heartbeat: Dietary polyphenols and vascular function
}

Epidemiological data suggests that a diet high in polyphenol-rich foods, such as berries and dark chocolate, is associated with lower cardiovascular disease risk. ${ }^{12}$ However, it remains unclear if this association reflects a cause-and-effect or whether it is simply due to associated clinical factors. The aim of the Polyphenol Intervention Trial (PPhIT) was to determine if increasing overall polyphenol dietary intake results in improved microvascular function, measured by forearm blood flow (FBF) responses to acetylcholine (Ach), an endothelium-dependent vasodilator (see page 1371). Hypertensive patients (51 per group) were randomized to a high or low polyphenol diet for 8 weeks. The high polyphenol group consumed a daily diet with 6 portions of fruits and vegetables, including one serving of berries, and $50 \mathrm{~g}$ of dark chocolate; whereas the low-polyphenol group was asked to avoid these foods. The high polyphenol group had larger increases in plasma vitamin C, serum carotenoids, and plasma epicatechin levels which suggests participants were compliant with the study diet. Forearm blood flow (figure 1) showed a significantly greatly vasodilator response in the high polyphenol group after intervention both when compared to baseline and when compared to the low polyphenol group.

Although many questions about the association between dietary polyphenols and cardiovascular risk remain unanswered, this small well-designed study does provide some insight into possible mechanisms by which polyphenol consumption might lower cardiovascular risk. In the accompanying editorial, Lamuela-Raventos and Quifer-Rada (see page 1340) suggest "Lifestyle and dietary interventions minimise dependency on pharmacological antihypertensive therapies, thus avoiding the adverse effects and costs of drug use. On the other hand, however delightful it is to consume berries and $50 \mathrm{~g}$ of dark chocolate daily, this amount of chocolate also contains about 300 calories; while benefits are seen with an 8-week diet, increased adiposity

Correspondence to Professor Catherine M Otto, Division of Cardiology, University of Washington, Seattle,WA 98195, USA; cmotto@u.washington.edu with long term consumption might mitigate any potential benefit.

Obesity is a recognized risk factor for atrial fibrillation (AF). However, recent studies "support the idea that cardiometabolic dysfunction is a better predictor of recurrent cardiovascular events than body

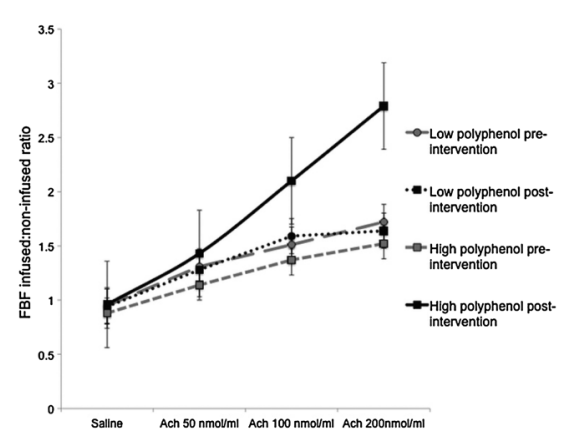

Figure 1 Forearm blood flow (FBF) doseresponse curves (ratio of infused:non-infused with error bars indicating standard error of mean) during saline and increasing concentrations of acetylcholine (Ach) for high-polyphenol and low-polyphenol diet preintervention and postintervention. Change in low-polyphenol group 12.8 (-34.8 to 60.4) versus change in high-polyphenol group 115.9 (70.1 to 161.6) $(p=0.02)$. Based on 92 subjects due to missing microvascular function data on one patient in the low-polyphenol group. mass index (BMI) per se." 3 Possible mechanisms for the association between BMI and cardiovascular events include secondary effects of comorbidities (e.g. hypertension, insulin resistance, etc) or primary mechanisms such as increased inflammation or altered adiocyte secretion of cytokines such as leptin, adiponectin and resistin. In order to address the mechanism of the association between body mass index (BMI) and AF, Ermakov and colleagues (see page 1354) examined data from a population based study of 4937 ethnically diverse women age 50-79 years with incident $\mathrm{AF}$ in $18 \%$ over a mean follow of 11.1 years. Although baseline levels of leptin, adiponectin and resistin were higher in women who developed AF, after multivariable adjustment only log resistin levels remained associated with incident $\mathrm{AF}(\mathrm{HR}=1.57$ per $1 \mathrm{log}(\mathrm{ng} / \mathrm{mL})$ increase, $p=0.006)$ and this association was only partially attenuated by adjustment for BMI and other inflammatory cytokines (figure 2).

In an editorial comment, Güngör (see page 1339) concludes "It is clear that adipokines have important role in human metabolism and abnormalities of adipose tissue cause metabolic abnormalities through adipokines." "Especially, the effect of gender and dietary habits should be considered in future studies and results

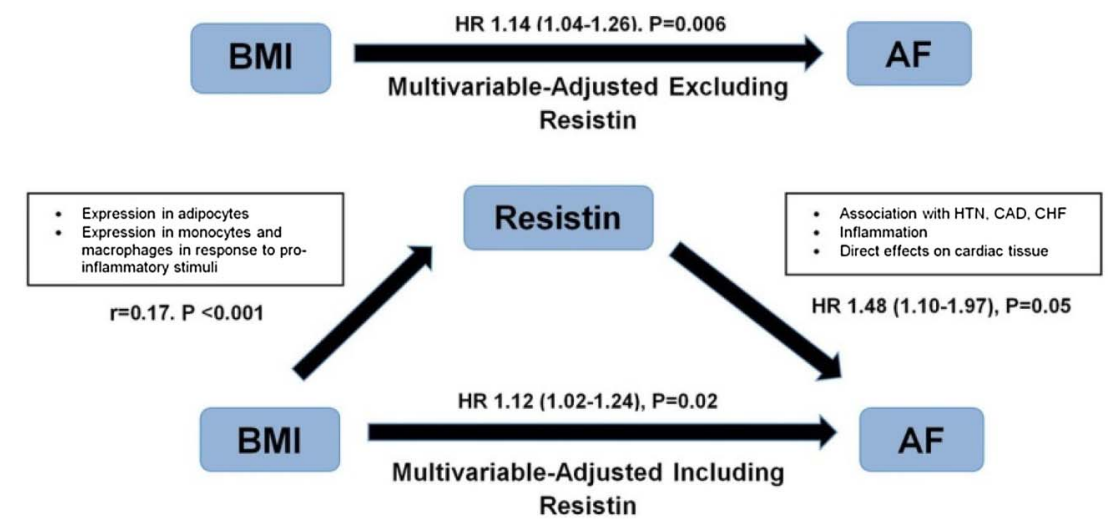

Figure 2 Body mass index (BMI)-atrial fibrillation (AF) mediation by resistin. BMI was significantly associated with AF upon multivariable adjustment excluding resistin. Introducing resistin into the model resulted in a decrease in AF risk suggesting partial mediation of the relationship. BMI was positively correlated with levels of resistin. In addition to the direct secretion of resistin from adipocytes, resistin expression in monocytes and macrophages may be unregulated in response to the inflammatory state of obesity. Elevated resistin on its own was significantly associated with increased AF risk. This relationship may be mediated through associated comorbidities (ie, hypertension (HTN), coronary artery disease (CAD), congestive heart failure (CHF)), its association with inflammation or its direct effects on cardiac tissue properties. 

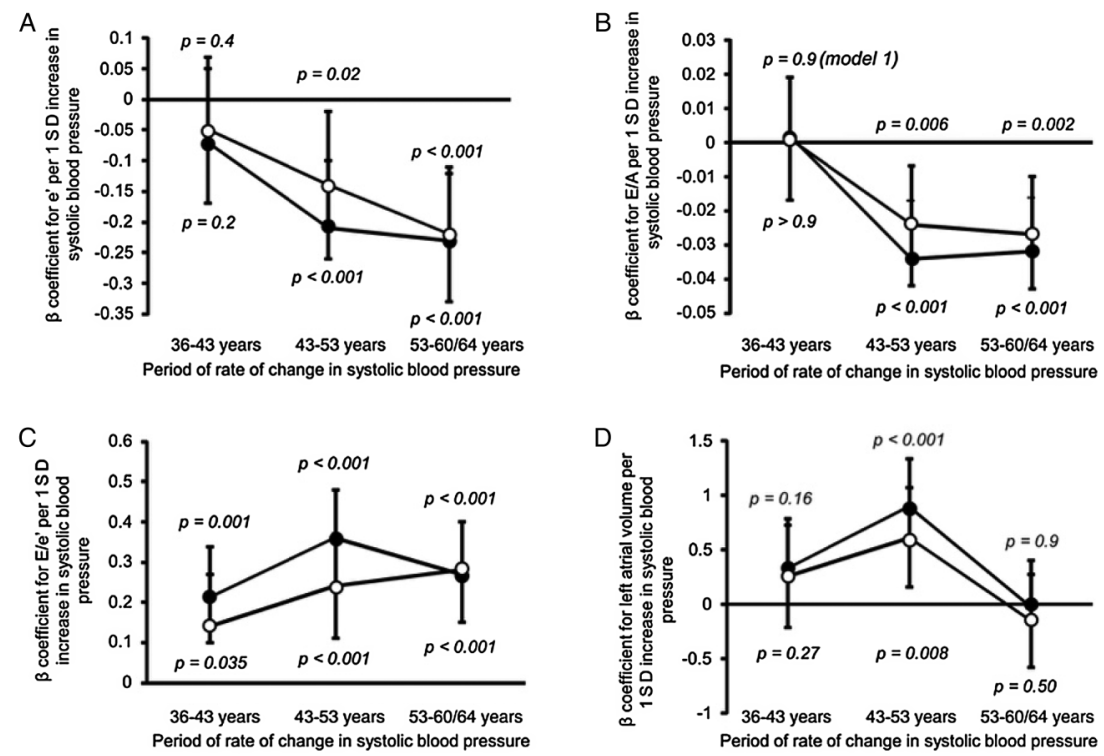

Figure 3 Association between standardised residuals of change in systolic blood pressure (SBP) over three time periods (36-43 years), (43-53 years) and (53-60 to 64 years) conditional on the earlier measure(s) and markers of diastolic function at age 60-64 years. The residuals can be interpreted as the standardised rate of change in SBP in an individual above or below that expected on average in the sample given their earlier SBP. (A) e $e^{\prime}$ at age 60-64 years $(n=1172)$. (B) E/A at age 60-64 years $(n=1190)$. (C) E/e' at age 60-64 years $(n=1120)$. (D) Left atrial volume indexed to body surface area at age $60-64$ years $(n=1054)$. Data points are $\beta$ coefficients and the bars represent $95 \%$ Cls. Model $1(\bullet)$ : adjusted for age, sex and clinical research facility attended. Model 2 (O): model 1+diabetes mellitus+body mass index+smoking status+physical activity status+current antihypertensive treatment. $p$ Values were calculated using Wald tests and $p<0.05$ indicates a significant association between the rate of change in SBP in the specified period and the measure of diastolic function.

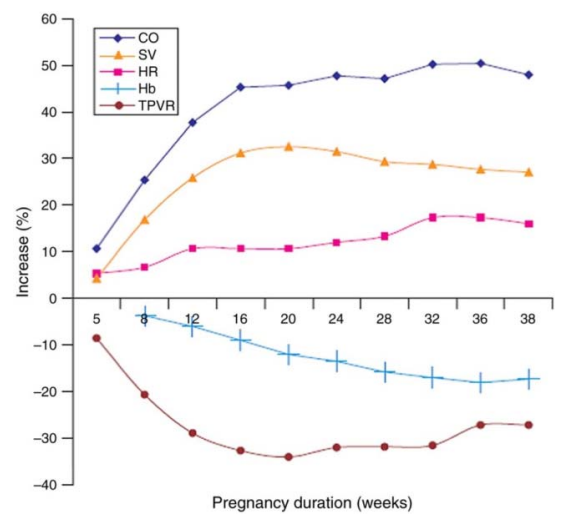

Figure 4 Plot showing the changes in cardiac output (CO), stroke volume (SV), total peripheral vascular resistance (TPVR), heart rate $(H R)$ and haemoglobin concentration $(\mathrm{Hb})$ during pregnancy. Reproduced from: Karamermer and Roos-Hesselink. ${ }^{4}$ of gender specific subgroup analysis should be included."

Heart failure with preserved ejection fraction, typically characterized by left ventricular (LV) diastolic dysfunction, is associated with a diagnosis of hypertension. However, the effects of elevated blood pressure (BP) over the patient's lifetime, versus current $\mathrm{BP}$, has not been defined.

In a cohort of 1653 adults, Ghosh and colleagues (see page 1380) found that echocardiographic parameters of diastolic dysfunction and elevated LV filling pressures measured at age 60-64 years, were better predicted by BP earlier in life than the BP or severity of LV hypertrophy at the time of the echocardiographic study (figure 3). The authors conclude: "BP from the age of 36 years predicts diastolic function in people aged 60-64 years independently of current BP (for $\mathrm{E} / \mathrm{e}^{\prime}$ and $\mathrm{LAVI})$; faster increases in BP in midlife are particularly detrimental. People on antihypertensive treatment have more adverse diastolic function even when current BP is taken into account suggesting that early risk factor modification may be important to prevent the adverse effects of $\mathrm{BP}$ on diastolic function".

The Education in Heart article in this issue is titled "Pregnancy and native heart valve disease" authored by Dr. Sara A Thorne (see page 1410). Learning objectives for this article are to understand the hemodynamic effects of pregnancy, to know how to manage women with native valve disease before and during pregnancy, to recognize that native valve regurgitation is better tolerated than valve stenosis during pregnancy and to be able to manage pregnancy in women with aortic or mitral valve stenosis figure 4.

The Image Challenge (see page 1387) in this issue shows a contrast enhanced CT scan of the abdomen after cardiac catheterization in a 57 year old woman.

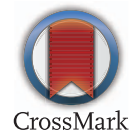

To cite Otto CM. Heart 2016;102:1337-1338.

Heart 2016;102:1337-1338.

doi:10.1136/heartjnl-2016-310315

\section{REFERENCES}

1 Kwok CS, S Boekholdt M, Lentjes MAH, et al. Habitual chocolate consumption and risk of cardiovascular disease among healthy men and women. Heart 2015;101:1279-87.

2 Larsson SC, Agneta Åkesson A, Bruna Gigante B, et al. Chocolate consumption and risk of myocardial infarction: a prospective study and meta-analysis. Heart 2016;102:1017-22.

3 Beleigoli A, de Fatima Diniz M. Two (or more) sides of a coin. Heart 2014;15;100:1399-401.

4 Karamermer Y, Roos-Hesselink JW. Pregnancy and adult congenital heart disease. Expert Rev Cardiovasc Ther 2007;5:859-69. 